\title{
Die Tuberkulosesituation in Deutschland 2000
}

\author{
R. Loddenkemper ${ }^{1}$ \\ A. Brendel ${ }^{1}$ \\ D. Sagebiel ${ }^{1}$ \\ M. Forßbohm²
}

The State of Tuberculosis in Germany in 2000

\section{Zusammenfassung}

Die Tuberkulose ist neben HIV/AIDS und Malaria eine der führenden Infektionskrankheiten, an der weltweit jährlich über 8 Millionen Menschen erkranken und schätzungsweise 2 Millionen versterben. Eine besondere Bedeutung für Deutschland hat die epidemiologische Entwicklung in der ehemaligen Sowjetunion, wo die Neuerkrankungszahlen stark ansteigen. Hier sind auch Brennpunkte bei der Entwicklung multiresistenter Tuberkulosen zu verzeichnen. Die WHO registrierte 2000 weltweit bereits 273000 Fälle multiresistenter Tuberkulosen.

In Deutschland ist die Tuberkulose-Situation stabil. Im Jahr 2000 erkrankten insgesamt 9064 Personen (910 weniger als 1999) an Tuberkulose. Auf die Tuberkulose der Atmungsorgane mit Nachweis von Tuberkulosebakterien entfielen 5271 Fälle, bei 2264 Tuberkulosen der Atmungsorgane wurden keine Tuberkulosebakterien nachgewiesen. 1529 Personen erkrankten an extrapulmonaler Tuberkulose. Die höchsten Erkrankungszahlen wurden bei der älteren Bevölkerung verzeichnet. Ein Drittel der Tuberkulosepatienten in Deutschland wurde im Ausland geboren.

Die Resistenzen gegen Antituberkulotika stiegen auch in Deutschland leicht an. In einer DZK-Studie wurde, bei allerdings kleinen Fallzahlen, ein Anstieg von 1,2 \% (1996) auf 1,7 \% (2000) registriert. Dies hatte Einfluss auf die Behandlungsergebnisse. Mit zunehmender Resistenz sank der Behandlungserfolg von $77,5 \%$ bei voll sensibler auf 59,5\% bei multiresistenter Tuberkulose.

\section{Abstract}

Tuberculosis is one of the leading infectious diseases globally besides HIV/AIDS and malaria. Around 8 million people per year develop active tuberculosis, of whom 2 million eventually die of the disease.

Of special importance to Germany is the epidemiologic situation in the former Soviet Union, where new infections are rising steeply. This region is also a hot spot for the development of multidrug-resistant tuberculosis. In 2000, the WHO registered already 273000 cases of multidrug-resistant tuberculosis worldwide.

In Germany the tuberculosis situation is stable. 9064 persons (910 less than 1999) had tuberculosis in the year 2000. 5271 cases were tuberculosis of the respiratory tract with detection of Mycobacteria tuberculosis. In 2264 cases registered as tuberculosis of the respiratory tract, Mycobacteria tuberculosis could not be detected. 1529 persons developed extrapulmonary tuberculosis.The highest incidences were found among the elderly. One third of the tuberculosis patients were born outside Germany.

Resistance to antituberculous drugs also increased slowly in Germany. A study of the DZK, although with small numbers, demonstrated an increase of multidrug-resistant tuberculosis from $1.2 \%$ (1996) to $1.7 \%$ (2000), which also influenced the treatment outcome. With rising resistance rates, the treatment success decreased from $77.5 \%$ in fully susceptible to $59.5 \%$ in multidrug-resistant cases.

${ }^{1}$ Deutsches Zentralkomitee zur Bekämpfung der Tuberkulose (DZK), Lungenklinik Heckeshorn, Berlin

${ }^{2}$ Gesundheitsamt Wiesbaden

Widmung

Die statistischen Daten wurden freundlicherweise vom Statistischen Bundesamt Wiesbaden und den statistischen Landesämtern zur Verfügung gestellt. Die DZK-Studienergebnisse zur Resistenz wurden von Herrn Dr. Forßbohm/Gesundheitsamt Wiesbaden in Zusammenarbeit mit 285 Gesundheitsämtern in Deutschland erhoben. Resistenzdaten wurden uns auch vom Arbeitskreis Mykobakterien (Hr. Dr. Küchler) sowie von Frau Dr. Rüsch-Gerdes vom Nationalen Referenzzentrum in Borstel zur Verfügung gestellt. Wir bedanken uns ganz herzlich für das Engagement und den Arbeitseinsatz aller Beteiligten. Unser besonderer Dank gilt dem Bundesministerium für Gesundheit für die Unterstützung des DZK.

Korrespondenzadresse

Prof. Dr. R. Loddenkemper · Generalsekretär · Deutsches Zentralkomitee zur Bekämpfung der Tuberkulose · Lungenklinik Heckeshorn · Zum Heckeshorn 33 · 14109 Berlin · E-mail: loddheck@zedat.fu-berlin.de 
Einleitung

Die Tuberkulose bleibt weiterhin bei Jugendlichen und Erwachsenen weltweit die am häufigsten zum Tode führende Infektionskrankheit. Die Weltgesundheitsorganisation (WHO) schätzt die Zahl der jährlichen Todesfälle auf 2-3 Millionen. Es wird davon ausgegangen, dass ein Drittel der Weltbevölkerung mit dem Tuberkulosebakterium infiziert ist. Etwa 5-10\% der Infizierten entwickeln im Laufe ihres Lebens eine aktive Tuberkulose. Die Zahl der jährlichen Neuerkrankungen wird weltweit auf insgesamt 8,7 Millionen geschätzt, wobei über 95\% aller Tuberkulosefälle in den so genannten Entwicklungsländern auftreten [18]. Die 22 Länder mit den meisten Tuberkulose-Erkrankungen verzeichneten im Jahr 2000 allein $80 \%$ aller Fälle. Neben Faktoren wie Armut, Mangelernährung, schlechter medizinischer Versorgung und Migration trägt insbesondere die HIV-Epidemie wesentlich zu einer Verschlechterung der weltweiten Tuberkulosesituation bei $[17,18]$.

Für Deutschland ist aufgrund der geografischen Nähe die Entwicklung in Osteuropa und der ehemaligen Sowjetunion von besonderer epidemiologischer Bedeutung [14]. Die Zunahme der Tuberkulosezahlen setzte sich in den Neuen Unabhängigen Staaten (NUS) weiter fort. Aber auch die Mortalität, ein Indikator für die Qualität der Tuberkulosetherapie, ist deutlich angestiegen $[7,11,14]$.

Aus Osteuropa werden gegenwärtig eine Viertelmillion neuer Tuberkulosefälle pro Jahr berichtet. Die höchste Rate an Neuerkrankungen wurde für 2000 - mit weiterhin steigender Tendenz aus den NUS, und hier insbesondere aus Kasachstan (160/100000), gemeldet [18]. Die rapide wachsende Zahl der HIV-Infektionen, besonders in Russland, gibt zusätzlichen Anlass zur Besorgnis $[8,11,17]$.

Ein besonders schwer wiegendes Problem ist die weltweite $\mathrm{Zu}$ nahme medikamentenresistenter Tuberkulosestämme. Auch hier sind die Nachfolgestaaten der Sowjetunion verstärkt betroffen. Insbesondere in Gefängnissen und ähnlichen Einrichtungen ist die Lage sehr ernst $[4,8,18]$.

Ursächlich für die Entwicklung von Resistenzen ist in erster Linie eine inadäquate Tuberkulosetherapie, bedingt durch fehlerhafte Verordnungen und/oder durch den Mangel an Medikamenten. Auch die ungenügende Kontrolle der Therapie sowie eine mangelhafte Patientenmitarbeit begünstigen die Entstehung von Resistenzen [10].

Der WHO wurden 2000 bereits 273000 Fälle von multiresistenter Tuberkulose gemeldet. Schätzungen gehen von 50 Millionen Menschen aus, die weltweit mit multiresistenten Bakterienstämmen (MDR-TB = multidrug-resistant tuberculosis, d.h. Resistenz gegen mindestens INH und RMP) infiziert sind [15].

\section{Die Tuberkulosesituation in der Bundesrepublik Deutschland 2000}

Im Folgenden werden die Daten des 27. Informationsberichtes des DZK für das Jahr 2000 unter besonderer Berücksichtigung der bekannten Risikopopulationen in Deutschland dargestellt [1].
9064 neue Erkrankungsfälle wurden in der Bundesrepublik Deutschland im Jahr 2000 registriert. Bei einer Gesamtbevölkerung von 82163475 Menschen entspricht dies einer Inzidenz von 11,0 pro 100000 Einwohner. Damit traten gegenüber 1999 910 Fälle weniger auf (-9,1\%). Den Verlauf der Tuberkuloseneuerkrankungen für die einheimische und die ausländische Bevölkerung seit 1974 zeigt Abb.1. Nur in den Jahren 1992 und 1993 war ein geringer Anstieg der Gesamtinzidenz zu verzeichnen. Der Hauptgrund für diesen Anstieg war die Zunahme der Tuberkuloseerkrankungen in der ausländischen Bevölkerung, da in diesem Zeitraum der Zustrom von Migranten besonders ausgeprägt war. Die Abb. 2 u. 3 zeigen die Anzahl und Inzidenzen Erkrankter von 1991 bis 2000. 33,6\% aller TB-Fälle traten bei der ausländischen Bevölkerung auf (1999: 33,1\%) (Abb.4).

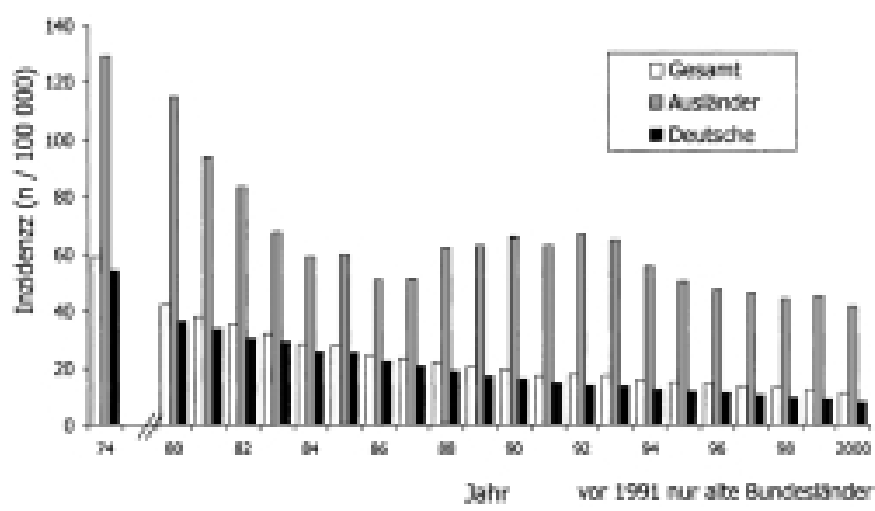

Abb. 1 Entwicklung der Tuberkuloseinzidenz in Deutschland 1974-2000.

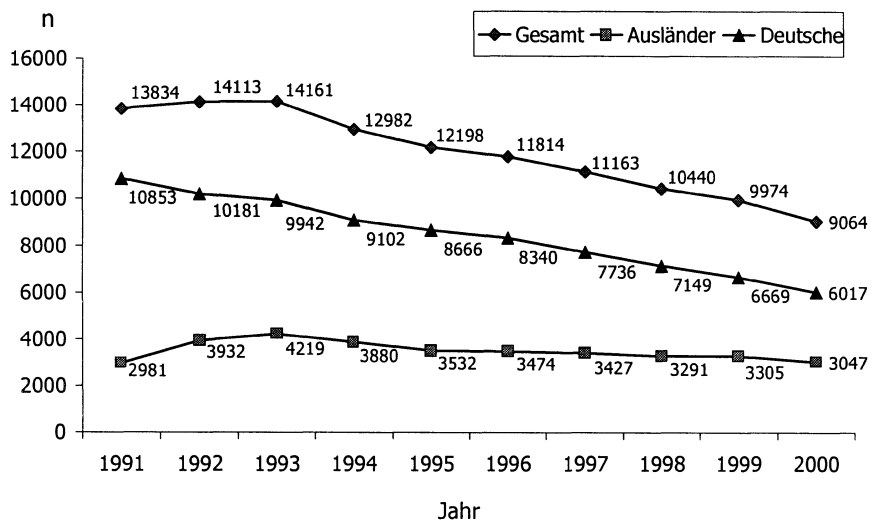

Abb. 2 Neuerkrankungen an Tuberkulose (Absolutzahlen) in Deutschland $(1991-2000)$.

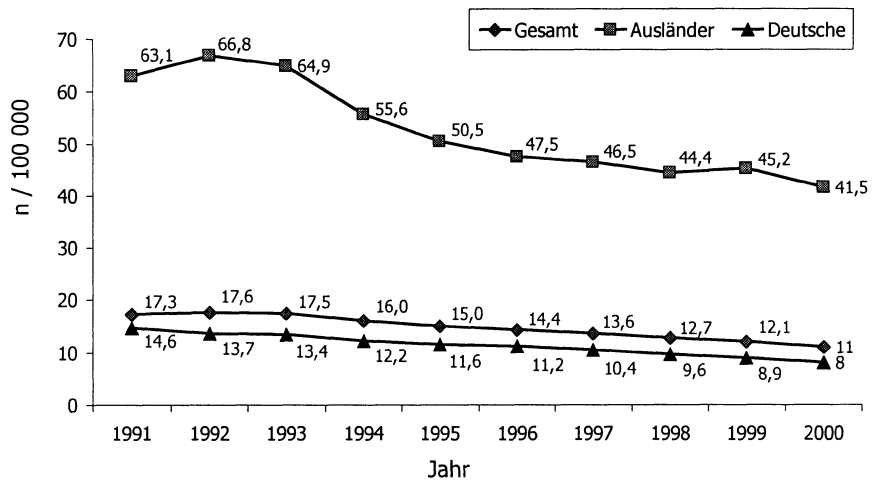

Abb. 3 Neuerkrankungen an Tuberkulose (Inzidenz) in Deutschland (1991-2000). 


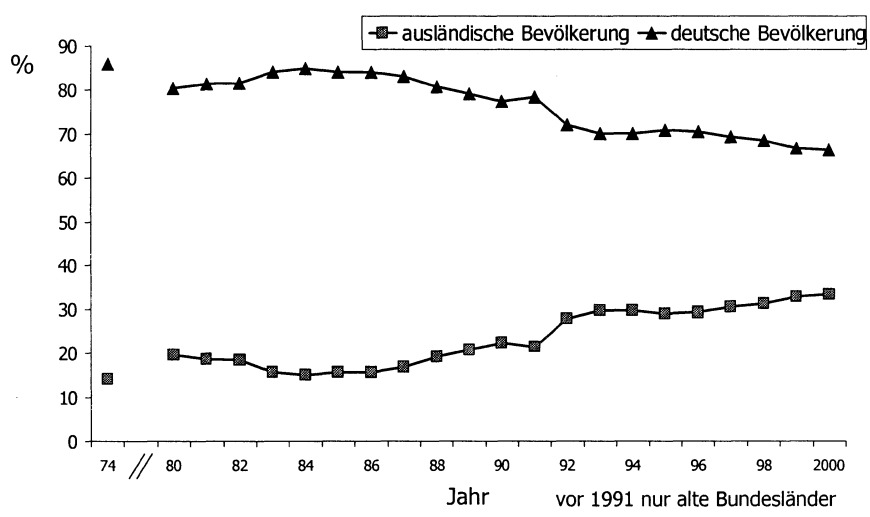

Abb. 4 Entwicklung der Tuberkulose in Deutschland 1974-2000; Anteil der deutschen und der ausländischen Bevölkerung.

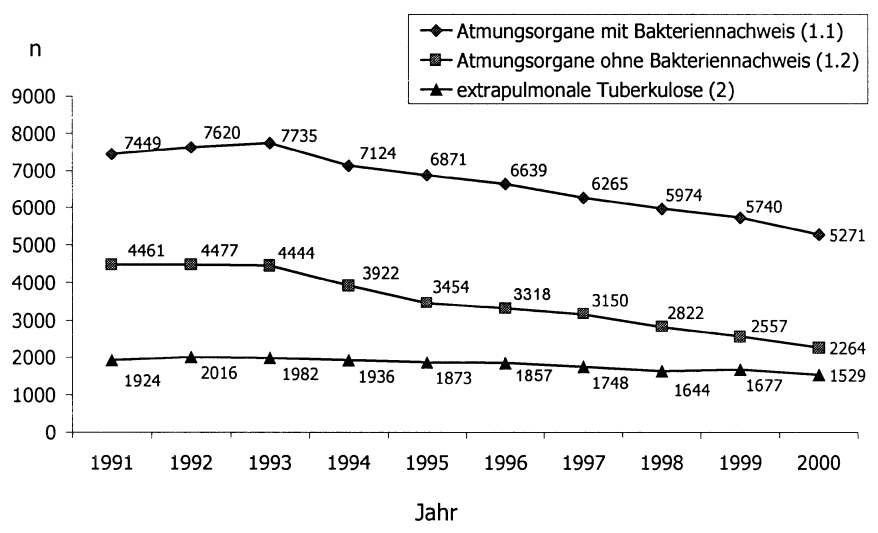

Abb. 5 Neuerkrankungen an Tuberkulose (Absolutzahlen) in Deutschland nach Diagnosegruppen (1991-2000).

Im Vergleich zu den Vorjahren hat sich die Verteilung auf die einzelnen Tuberkuloseformen nicht wesentlich verändert: Auf Tuberkulose der Atmungsorgane mit Nachweis von Tuberkulosebakterien (Diagnosegruppe 1.1) entfielen 5271 Fälle (58,2\%), auf Tuberkulose der Atmungsorgane ohne Nachweis von Tuberkulosebakterien (Diagnosegruppe 1.2) 2264 Fälle (25,0\%). Auf Tuberkulose anderer Organe (Diagnosegruppe 2) entfielen dagegen 1529 Fälle (16,9\%) (Abb. 4 u. 5). Unter den 5271 offen-tuberkulösen Fällen gelang bei 2665 (50,6\%) der direkte Nachweis von Tuberkulosebakterien im Sputum. Dies ist der epidemiologisch entscheidende, da besonders ansteckungsfähige, Anteil an allen Tuberkuloseformen.

Der weiterhin fortgesetzte Rückgang an Neuerkrankungen ist durch die Abnahme aller Tuberkuloseformen bedingt. Die Zahl der offenen Tuberkuloseformen ist im Vergleich zu 1999 um $8,2 \%$, die der geschlossenen Tuberkuloseformen um 11,5\% zurückgegangen. Im Gegensatz zu 1999 zeigte sich bei den extrapulmonalen Tuberkulosen ebenfalls ein Rückgang um 8,8\%.

Wie auch in den Vorjahren erkrankten Männer nahezu doppelt so häufig an Lungentuberkulose wie Frauen (65,6\% vs. $34,4 \%$ ), die Inzidenz der Frauen betrug 6,2/100000 Einwohner, die der Männer 12,3/100 000.

Bei den extrapulmonalen Tuberkuloseformen erkrankten Frauen weiterhin etwas häufiger (55,8\%). Die Verteilung innerhalb der extrapulmonalen Formen stellt sich im Wesentlichen unverän-

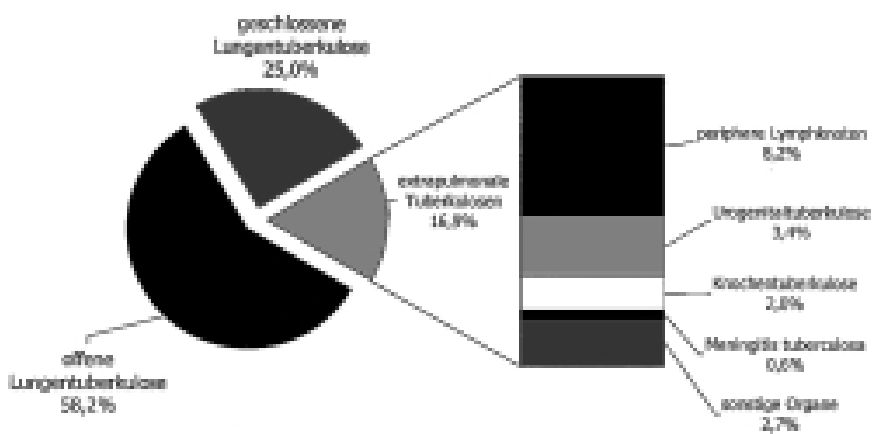

Abb. 6 Verteilung der Tuberkuloseformen in Deutschland 2000 ( $\mathrm{n}=9064)$.

dert dar. Am häufigsten wurde die periphere Lymphknotentuberkulose mit 48,5\% beobachtet, gefolgt von der Urogenitaltuberkulose mit 20,4\%. Bei 11,8\% der Fälle waren Knochen und Gelenke betroffen, eine Meningitis tuberculosa fand sich in 3,3\% der Fälle (51 Personen, darunter drei einheimische und sechs ausländische Kinder), und 16,0\% der extrapulmonalen Tuberkulosen entfielen auf sonstige Organe (Abb. 6).

\section{Die Altersverteillung der Tuberkuloseerkrankungen}

Abb. 7 zeigt die Tuberkuloseinzidenzen bezogen auf Alter und Geschlecht. Die Inzidenz der Frauen für alle Tuberkuloseformen betrug 8,2/100 000, die der Männer 14,0/100000. An Tuberkulose erkrankten unverändert am häufigsten ältere Männer. Männer, die älter als 75 Jahre waren, zeigten die höchste Inzidenz, an Tuberkulose zu erkranken (39,7/100 000). Inzidenz und Mortalität in Abhängigkeit von den einzelnen Altersgruppen werden in Abb. 8 gezeigt.

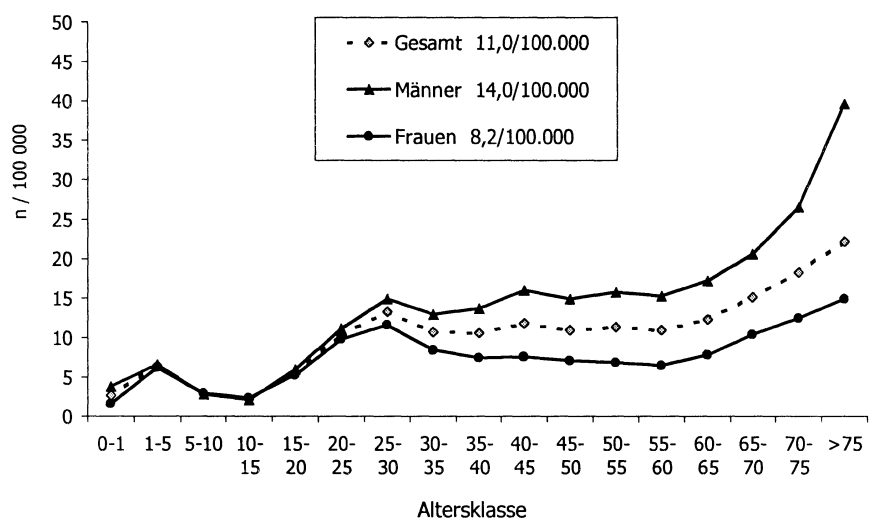

Abb. 7 Tuberkuloseinzidenzen (alle Formen) in Deutschland 2000 nach Alter und Geschlecht.

Abb. 9 zeigt die Altersverteilung für die einheimische und die ausländische Bevölkerung. Bei der einheimischen Bevölkerung nimmt die Inzidenz nahezu kontinuierlich mit höherem Alter zu, während sich bei der ausländischen Bevölkerung drei Inzidenzgipfel finden: Kleinkinder (1 - 5 Jahre), jüngere Erwachsene (20-45 Jahre) und über 65-Jährige zeigen im Vergleich zu den anderen Altersgruppen deutlich erhöhte Inzidenzen (die altersabhängigen Inzidenzraten sind in Abb.9 als unstandardisierte Inzidenzraten für die einzelnen Altersgruppen abzulesen). 


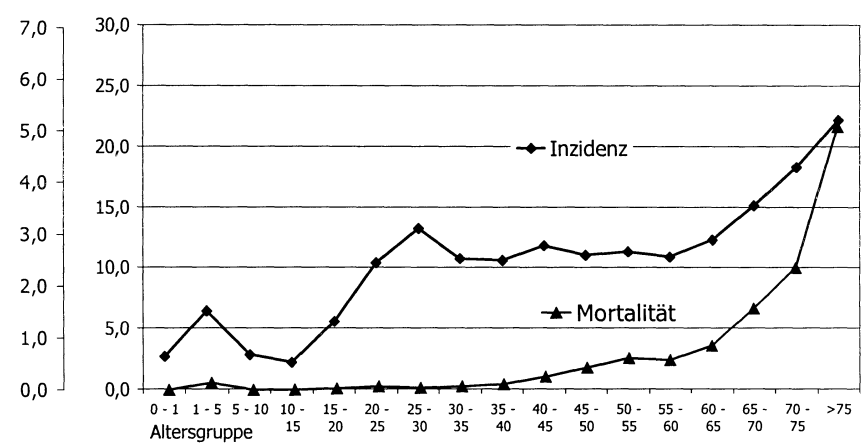

Abb. 8 Neuerkrankung und Sterblichkeit für alle Tuberkuloseformen in Deutschland 2000.

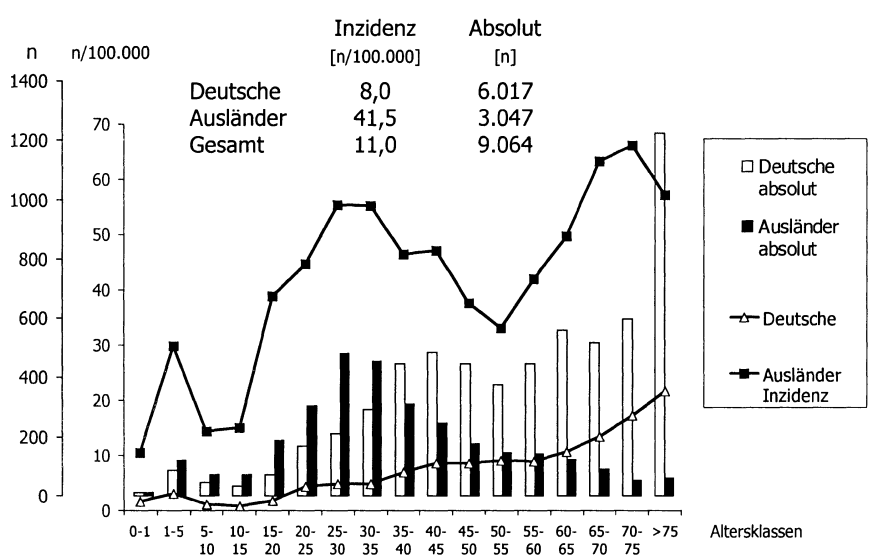

Abb. 9 TB-Fälle in Deutschland (Inzidenzen und Absolutzahlen) nach Alter und Herkunft 2000.

\section{Die Mortalität der Tuberkulose}

2000 starben in der Bundesrepublik Deutschland 607 Menschen an einer Tuberkulose oder deren Spätfolgen. Dies waren 3,7\% weniger als 1999 (630 Sterbefälle). Den „primären Phthisentod“ starben 497 Personen, während 110 Personen an den Spätfolgen einer Tuberkulose verstarben. Der prozentuale Anteil der Sterbefälle an aktiver Tuberkulose war mit 5,5\% gegenüber den Vorjahren leicht angestiegen (1999: 5,0\%). Die Mortalität der Tuberkulose lag 2000 bei 0,74 pro 100000 Einwohner und war somit etwas geringer als im Vorjahr (1999: 0,77/100000; Tab. 1, Abb. 8).

\section{Die Tuberkulosesituation in den einzelnen Bundesländern 1998-2000}

Im Jahr 2000 waren die Tuberkuloseinzidenzen überwiegend rückläufig. Allein im Saarland wurde ein Anstieg der Inzidenz von 12,0 (1999) auf 13,8 verzeichnet. In Hamburg war die Inzidenz - nach dem Anstieg im Jahre 1999 (19,1) - mit 17,2 wieder nahezu auf dem Stand von 1998 (16,8). Hamburg, Hessen, Berlin, das Saarland, Nordrhein-Westfalen und Bremen lagen über dem Bundesdurchschnitt von 11,0/100 000. Bei den Erkrankungen an offener TB lag neben den aufgeführten Ländern auch Mecklenburg-Vorpommern über dem Bundesdurchschnitt von 6,4.
Tab. 1 Neuerkrankungen und Sterbefälle an Tuberkulose $1993-2000$

\begin{tabular}{|lllll|}
\hline & $\begin{array}{l}\text { Tuberkulose- } \\
\text { Erkrankungen }\end{array}$ & $\begin{array}{l}\text { Sterbefälle an } \\
\text { aktiver Tuberkulose }\end{array}$ & $\begin{array}{l}\text { Sterbefälle einschließ- } \\
\text { lich Spätfolgen }\end{array}$ \\
\hline & $\mathrm{N}$ & $\mathrm{n}$ & $\%$ & $\mathrm{~N}$ \\
\hline 1991 & 13834 & 868 & 6,3 & 1146 \\
\hline 1992 & 14113 & 866 & 6,1 & 1155 \\
\hline 1993 & 14161 & 774 & 5,5 & 1037 \\
\hline 1994 & 14982 & 758 & 5,8 & 1014 \\
\hline 1995 & 12198 & 709 & 5,8 & 935 \\
\hline 1996 & 11814 & 692 & 5,8 & 896 \\
\hline 1997 & 11163 & 593 & 5,3 & 805 \\
\hline 1998 & 10440 & 541 & 5,2 & 711 \\
\hline 1999 & 9974 & 499 & 5,0 & 630 \\
\hline 2000 & 9064 & 497 & 5,5 & 607 \\
\hline
\end{tabular}

Die Länder mit den niedrigsten Gesamt-Inzidenzraten waren Sachsen (7,6), Brandenburg (7,9), Thüringen $(8,1)$ und SchleswigHolstein (8,2). In Abb. 10 sind die Inzidenzen der einzelnen Bundesländer von 1998 bis 2000 aufgeführt. Die Karte in Abb.11 zeigt die Kreise der Bundesrepublik mit Inzidenzen $>$ 13,8/100000 Einwohnern (20\% der Kreise mit den höchsten Tuberkuloseinzidenzen).

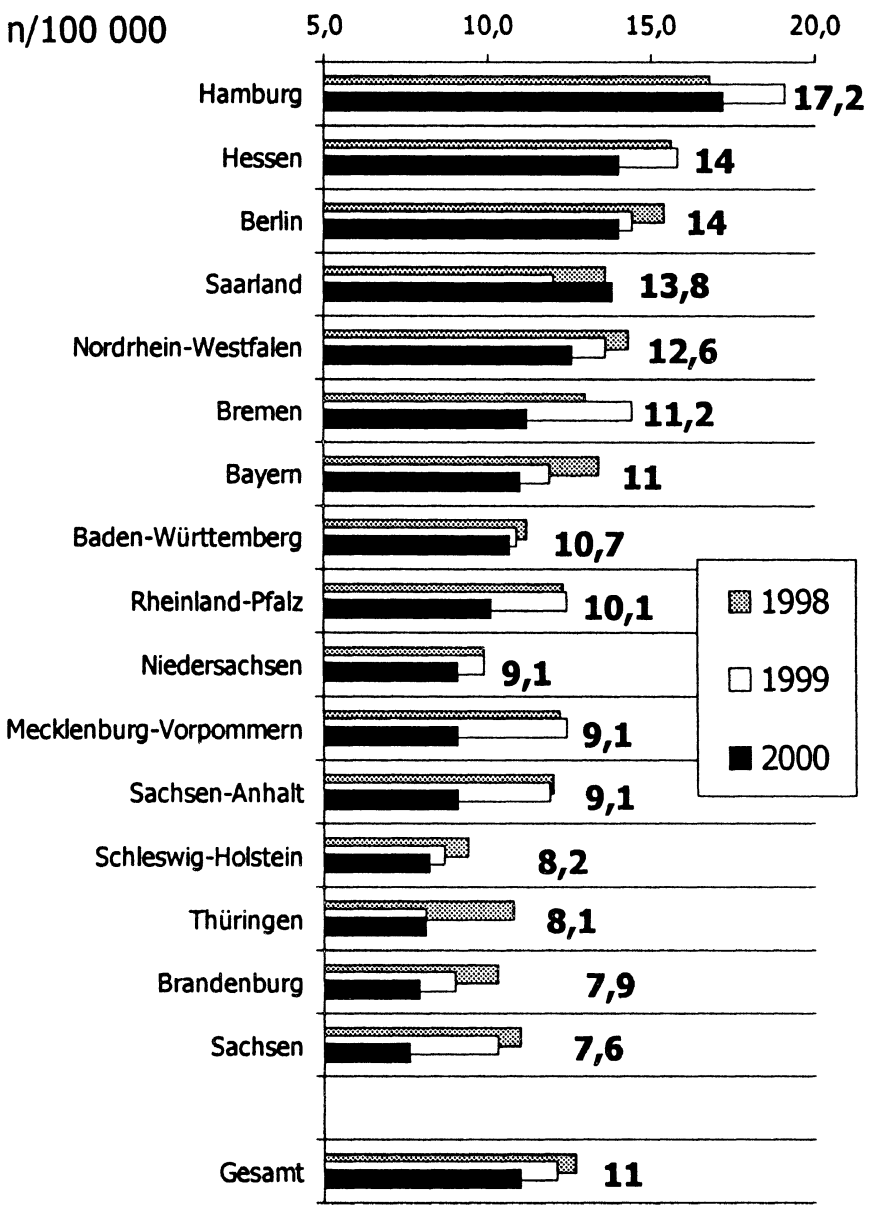

Abb. 10 Tuberkuloseinzidenzen (alle Formen, Gesamtbevölkerung) für die einzelnen Bundesländer 1998-2000. 


\section{Tbc-Neuzugänge 2000}

Pro 00.000 der Bevölkerung

Die obere Klasse beinhatet $20 \%$ der Kreise

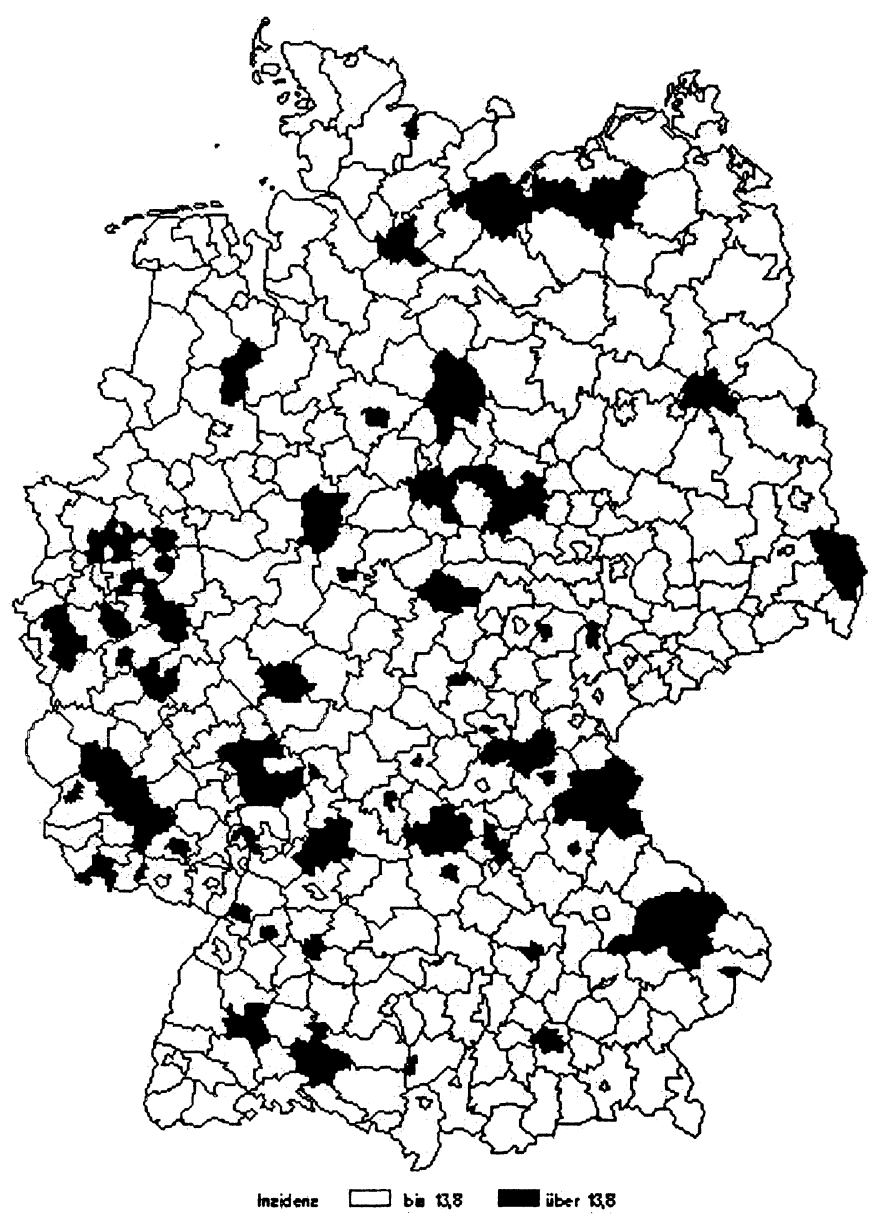

Abb. 11 Regionale Verteilung der Tuberkulose-Neuzugänge 2000 pro 100000 der Bevölkerung (Kreise mit Inzidenz > 13,8/100000).

Die Tuberkulose der in der Bundesrepublik lebenden Ausländer

In der Bundesrepublik Deutschland lebten am 31.12.2000 insgesamt 7296817 Ausländer (Ausländeranteil an der Gesamtbevölkerung 8,9\%) [16]. Bezüglich epidemiologischer Aussagen ist zu berücksichtigen, dass die Tuberkulosemeldestatistik, entgegen der DZK-Statistik, lediglich die Staatsangehörigkeit - und nicht das Geburtsland - berücksichtigt. Die Gruppe der Spätaussiedler wird somit aufgrund ihrer Staatsbürgerschaft zur deutschen Bevölkerung gezählt. Im Rahmen des Infektionsschutzgesetzes erfolgt seit 2001 auch eine Differenzierung nach Geburtsland.

Unter der ausländischen Bevölkerung waren bundesweit am 31.12.2000 Türken mit 1998534 am stärksten vertreten, gefolgt von Jugoslawen, Bosniern, Herzegowinern, Kroaten, Mazedoniern und Slowenen mit zusammen 1035616, Italienern mit 619060, Griechen mit 365438 und Polen mit 301366 [16]. Insgesamt stammten 80,3\% der in Deutschland lebenden Ausländer aus Europa (5857 791), 11,5\% aus Asien (841738), 4,1\% aus Afrika (299255), 2,9\% (213285) aus Amerika, 0,1\% aus Australien/ Ozeanien (10410) und 1,0\% waren staatenlos oder mit ungeklärter Staatsangehörigkeit (74338) [15,16].
2000 stellten 78564 Personen einen Antrag auf Asyl. Damit ist die Zahl der Antragsteller weiterhin rückläufig (vgl. 1999: 95113). Der größte Teil der Antragsteller kam aus Asien (39091; vor allem Irak) gefolgt von Osteuropa (18359; insbesondere aus dem ehemaligen Jugoslawien) sowie der Türkei (8968).

Unter den im Jahr 2000 an Tuberkulose Erkrankten befanden sich 3047 ausländische Mitbürger (33,6\%). Gegenüber 1999 waren dies 258 Personen weniger (-7,8\%), was einer Inzidenz von 41,5 pro 100000 entsprach. Damit lag die Inzidenz 5,2fach höher als die der einheimischen Bevölkerung (8,0/100 000). Für die einheimische Bevölkerung war im gleichen Zeitraum ein Rückgang der Neuerkrankungen von 652 Fällen zu verzeichnen (-9,8\%).

Die Inzidenz der Erkrankung an einer offenen Tuberkulose war 2000 für die in der Bundesrepublik lebenden Ausländer mit 21,1/100000 4,2fach höher als die der einheimischen Bevölkerung (5,0/100 000). 1549 Fälle ließen sich der Diagnosegruppe 1.1 zuordnen (50,8\%), 847 Fälle der Diagnosegruppe 1.2 (27,8\%), und 651 Fälle $(21,4 \%)$ fielen unter die Diagnosegruppe 2. Bei den Einheimischen betrug der Anteil offener Tuberkulosen 61,2\%, geschlossener Formen 23,5\% und extrapulmonaler Tuberkulosen $14,6 \%$. Auch in der ausländischen Bevölkerung erkrankten Männer nahezu doppelt so häufig an Lungentuberkulose wie Frauen, während extrapulmonale Tuberkulosen bei Frauen etwas häufiger auftraten.

\section{Die Tuberkulose im Kindesalter}

In der statistischen Erfassung werden Personen zum Kindesalter gerechnet, die das 15. Lebensjahr noch nicht vollendet haben. 2000 lebten insgesamt 12897014 Kinder in Deutschland [15,16].

In Deutschland erkrankten 2000 insgesamt 446 Kinder an einer aktiven Tuberkulose, dies entspricht einer Inzidenz von 3,46/100 000 Kinder (Abb. 12). Im Vergleich zu 1999 wurden somit 21 Fälle mehr gemeldet $(+4,7 \%)$. Dieser Anstieg ist vor allem auf eine Zunahme der Lymphknotentuberkulose bei den 1-5-jährigen Kindern zurückzuführen. Die Inzidenz stieg im Vergleich zu 1999 bei den in Deutschland lebenden ausländischen Kindern mit 18,6/100 000 leicht an und blieb bei den deutschen Kindern mit 1,5/100000 nahezu unverändert (Abb.12). Das Risiko ausländischer Kinder, an einer Tuberkulose zu erkranken, lag je nach Altersgruppe 7 -19fach höher als das deutscher Kinder.

Der größte Teil der 446 tuberkulosekranken Kinder (57,0\%) entfiel auch 2000 auf die Diagnosegruppe 1.2 (Tuberkulose der Atmungsorgane ohne Nachweis von Tuberkulosebakterien, $\mathrm{n}=254$ ); unter die Diagnosegruppe 1.1 (Tuberkulose der Atmungsorgane mit Nachweis von Tuberkulosebakterien, $\mathrm{n}=101$ ) fielen $22,6 \%$ und unter die Diagnosegruppe 2 (extrapulmonale Tuberkulosen) $20,4 \%$ der Fälle $(n=91)$.

Der Anteil offener Fälle (Diagnosegruppe 1.1) an der Gesamtzahl der Lungentuberkulosen (Diagnosegruppen 1.1 und 1.2) betrug 28,5\% (101/355), davon waren 62 ausländische Kinder (61,4\%). In 29,7\% der Fälle (30/101) gelang dabei der Nachweis von M. tuberculosis direkt im Sputumausstrich. Somit fand sich lediglich bei 


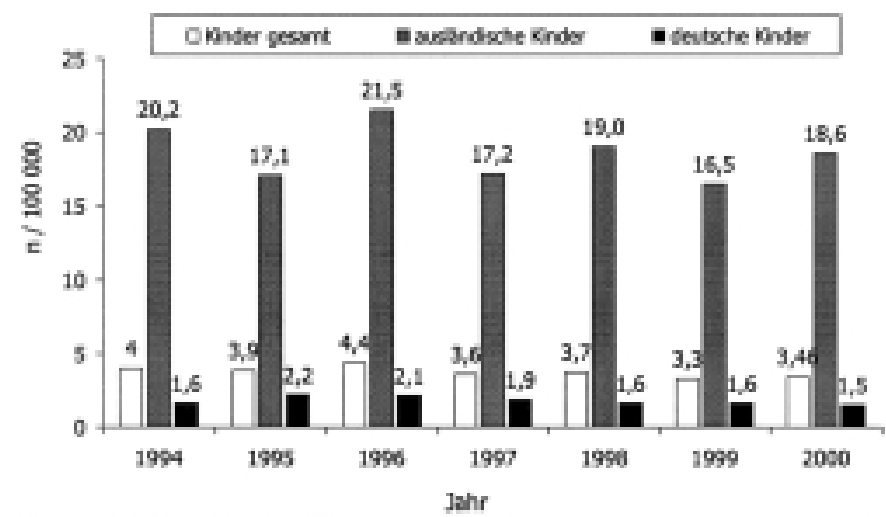

Abb. 12 Tuberkuloseinzidenzen beim Kind in Deutschland: Gesamt, ausländische und deutsche Kinder (1994-2000).

6,7\% aller an Tuberkulose erkrankten Kinder eine Lungentuberkulose mit positivem mikroskopischen Befund im Sputum (30/446).

Bei den extrapulmonalen Tuberkulosen (91 Fälle, davon 47 bei ausländischen Kindern) war auch 2000 die Tuberkulose der peripheren Lymphknoten mit 61 Fällen (67,0\%) am häufigsten, darunter waren 26 ausländische Kinder. Es folgte mit neun Fällen $(9,9 \%)$ die Meningitis tuberculosa. An einer Knochentuberkulose erkrankten acht Kinder (8,8\%), davon sechs ausländische. Drei Erkrankungen an einer Urogenitaltuberkulose wurden 2000 gemeldet. Eine Tuberkulose der "sonstigen Organe“ wurde in zehn Fällen berichtet, wobei hier die statistische Erfassung keine weitere Differenzierung (z.B. Miliartuberkulose) ermöglicht. Abb.13 zeigt die unterschiedliche Verteilung der Tuberkuloseformen für
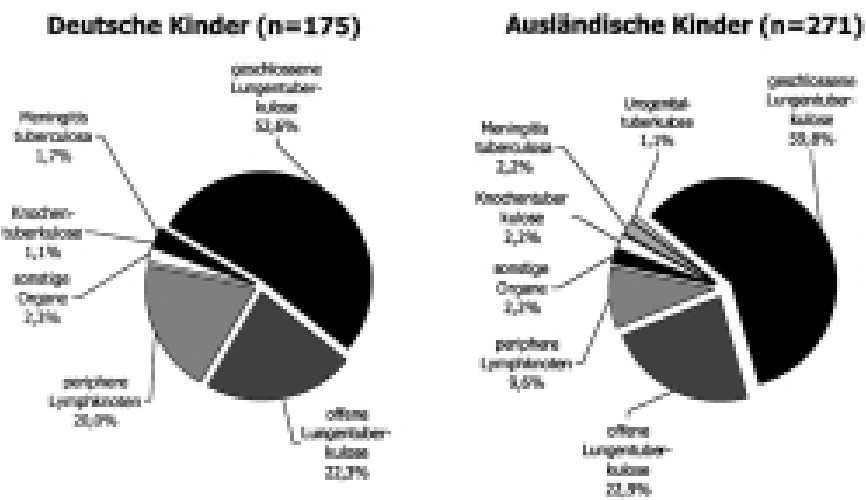

Abb. 13 Vergleich der Tuberkuloseformen bei ausländischen und einheimischen Kindern in Deutschland 2000.

einheimische und ausländische Kinder. Bei einheimischen Kindern wurden anteilig mehr Tuberkulosen der peripheren Lymphknoten beobachtet.

Wie oben bereits aufgeführt, erkrankten 2000 insgesamt neun Kinder an einer Meningitis tuberculosa (9,9\% der extrapulmonalen Tuberkulosen), darunter waren sechs Kinder ausländischer Herkunft. Die Inzidenz einer tuberkulösen Meningitis bei ausländischen Kindern betrug somit 0,41/100000 (1999: $0,34 / 100000$ ), die bei einheimischen Kindern 0,026/100000 (1999: 0,026/100000).
2000 lebten 1457453 ausländische Kinder in Deutschland (11,3\%) (Stichtag 31.12.1999) [15]. Von den insgesamt 446 erkrankten Kindern waren 271 ausländische Kinder (60,8\%). Die Inzidenz lag bei den 1-5-jährigen ausländischen Kindern mit 29,9/100 000 Kinder am höchsten (1999: 21,2/100000); während sie bei deutschen Kindern dieser Altersgruppe 3,1/100 000 betrug. Somit lag das Risiko ausländischer Kinder, an einer Tuberkulose zu erkranken, je nach Altersgruppe 7-19fach höher als das deutscher Kinder.

Aufgrund konstant niedriger Tuberkuloseinzidenzen und aktueller Nutzen-Risiko-Abwägungen wird von der Ständigen Impfkommission am Robert Koch-Institut (STIKO) die BCG-Impfung in Deutschland seit März 1998 nicht mehr empfohlen [12].

\section{Resistenz von M. tuberculosis-Stämmen in Deutschlland}

Im Folgenden sind die Ergebnisse der Umfrage des Arbeitskreises Mykobakterien (AKM) zu Empfindlichkeitsprüfungen von M.-tuberculosis-Komplex (Erstisolate) für 1991-2000 [1,7] im Vergleich mit den Daten der DZK-Studie zur Epidemiologie der Tuberkulose [1,2,15] dargestellt (Tab. 2, 3).

Die Ergebnisse der seit 1996 durchgeführten DZK-Studie zur Epidemiologie der Tuberkulose in Deutschland [5], an der 2/3 aller deutschen Gesundheitsämter teilnehmen und die etwa $60 \%$ aller Patienten in Deutschland erfasst, zeigen für 2000 einen Anstieg der Resistenz. Während sich in der deutschen Bevölkerung keine Zunahme der Resistenzen feststellen lässt, finden sich (multi-)resistente Stämme insbesondere bei der im Ausland geborenen Bevölkerung. Die höchste Resistenzrate gegenüber jeglichen Erstrangmedikamenten wurde, bei insgesamt kleinen Fallzahlen, mit $36,8 \%$ bei in den NUS geborenen Tuberkulosepatienten, die in Deutschland leben, beobachtet. Hauptsächlich traten hier Resistenzen gegenüber INH und SM auf $(27,8 \%$ und $31,1 \%)$, gefolgt von PTH (12,5\%), EMB (11,0\%) und RMP (9,0\%). Ein ähnliches Bild zeigt sich bei der MDR-TB, deren Rate 2000 für Patienten aus den NUS 8,5\% (1999: 9,4\%,1998: 11,2\%,1997: 11,8\%) betrug, während die MDR-TB in Deutschland insgesamt von 1,2\% (1996) auf 1,7\% (2000) anstieg $[1,5]$.

Die in den Vorjahren insbesondere bei der im Ausland geborenen Bevölkerung angestiegenen Resistenzraten verdeutlichen die Auswirkungen des weltweiten Anstiegs resistenter Tuberkulosestämme auch in Deutschland, wobei die geografische Nähe zu Osteuropa eine besondere Rolle spielt. Insbesondere die dramatische Entwicklung in den NUS lässt eine weitere Zunahme der Resistenzen dieser Personengruppe in Deutschland erwarten. Demgegenüber wurde in der DZK-Studie bei den in Deutschland Geborenen keine Zunahme der Resistenzen beobachtet [5]. Es ist daher notwendig, Patienten, die aus Ländern mit hohen Resistenzraten stammen und/oder bereits antituberkulotisch vorbehandelt wurden, bis zum Erhalt des Resultates der Empfindlichkeitsprüfung streng zu isolieren und initial mit vier, oder besser mit fünf, Medikamenten zu behandeln [3,6].

Auch in den Umfrageergebnissen des AKM wurde ein Anstieg resistenter Tuberkulosebakterien in Deutschland beobachtet. Die Angaben zur Resistenz gegen Pyrazinamid sind mit Vorbehalt zu bewerten, weil nicht genug Informationen über die Häufigkeit 
Tab. 2 Resistenzen gegen Antituberkulotika (DZK-Studie 1996-2000)

\begin{tabular}{llllllllll}
\hline Jahr & $\boldsymbol{n}$ & $\begin{array}{l}\boldsymbol{H} \\
\%\end{array}$ & $\begin{array}{l}\boldsymbol{R} \\
\%\end{array}$ & $\begin{array}{l}\boldsymbol{S} \\
\%\end{array}$ & $\begin{array}{l}\boldsymbol{E} \\
\%\end{array}$ & $\begin{array}{l}\boldsymbol{Z} \\
\%\end{array}$ & $\begin{array}{l}\boldsymbol{H}+\boldsymbol{R} \\
\%\end{array}$ & $\begin{array}{l}\text { \% jegliche Resistenz } \\
\text { (HRES) }\end{array}$ & $\begin{array}{l}\text { \% jegliche Resistenz } \\
\text { (HRESZ) }\end{array}$ \\
\hline 1996 & 2720 & 5,0 & 1,5 & 3,5 & 1,5 & 1,9 & $1,2(\mathrm{n}=33)$ & 6,8 & 7,9 \\
1997 & 3170 & 5,4 & 1,6 & 3,9 & 1,4 & 1,8 & $1,3(\mathrm{n}=42)$ & 7,3 & 8,6 \\
\hline 1998 & 3273 & 6,2 & 1,7 & 5,0 & 1,5 & 1,8 & $1,3(\mathrm{n}=44)$ & 8,9 & 10,4 \\
\hline 1999 & 3257 & 5,8 & 1,7 & 5,2 & 1,7 & 1,9 & $1,4(\mathrm{n}=45)$ & 8,5 & 9,9 \\
\hline 2000 & 2781 & 6,0 & 1,9 & 5,7 & 1,6 & 1,7 & $1,7(\mathrm{n}=48)$ & 8,6 & 10,3 \\
\hline
\end{tabular}

DZK-Studienergebnisse: alle kulturell gesicherten Tuberkulosen, für die mindestens das Ergebnis der Resistenzprüfung gegen INH und RMP bekannt war. „) Jegliche“ Resistenz wird in Bezug auf H, R, E oder S für internationale Vergleiche, bzw. H, R, Z, E oder S für Vergleiche mit AKM-Daten ausgewiesen. Doppelzählungen sind ausgeschlossen.

$H=I N H, R=R M P, S=S M, E=E M B, Z=P Z A$

Tab. 3 Resistenzen gegen Antituberkulotika (Umfrageergebnisse des AKM 1991 - 2000)

\begin{tabular}{|c|c|c|c|c|c|c|c|c|c|}
\hline \multicolumn{10}{|c|}{ AKM-Ergebnisse } \\
\hline Jahr & $\begin{array}{l}\text { Zahl der } \\
\text { teilnehm. Labore }\end{array}$ & $\begin{array}{l}\text { Zahl der } \\
\text { Stämme }\end{array}$ & $\begin{array}{l}\boldsymbol{H}_{r} \\
\%\end{array}$ & $\begin{array}{l}R_{r} \\
\%\end{array}$ & $\begin{array}{l}S_{r} \\
\%\end{array}$ & $\begin{array}{l}E_{r} \\
\%\end{array}$ & $\begin{array}{l}Z_{r}^{*} \\
\%\end{array}$ & $\begin{array}{l}H_{r}+R_{r}\left(+X_{r}\right) \\
\%\end{array}$ & $\begin{array}{l}\text { \% jegliche } \\
\text { Resistenz (HRSEZ) }\end{array}$ \\
\hline 1991 & 32 & 2776 & 5,1 & 1,4 & 2,9 & 1,0 & - & 1,1 & - \\
\hline 1992 & 32 & 3345 & 5,6 & 1,5 & 5,0 & 1,0 & - & 1,0 & - \\
\hline 1993 & 25 & 2654 & 6,3 & 1,7 & 5,0 & 1,9 & - & 1,4 & - \\
\hline 1994 & 27 & 2418 & 4,4 & 1,5 & 3,9 & 1,0 & - & 1,2 & - \\
\hline 1995 & 33 & 2579 & 5,5 & 1,6 & 4,7 & 2,0 & 1,4 & 1,3 & - \\
\hline 1996 & 40 & 3332 & 5,6 & 1,7 & 4,0 & 1,2 & 1,1 & 1,4 & - \\
\hline 1997 & 43 & 3267 & 7,1 & 2,5 & 4,7 & 2,3 & 1,7 & 2,1 & 10,5 \\
\hline 1998 & 28 & 2727 & 7,2 & 2,1 & 5,3 & 2,2 & 1,5 & 1,7 & 10,9 \\
\hline 1999 & 35 & 2907 & 6,4 & 2,1 & 5,5 & 1,6 & 1,4 & 1,9 & 9,4 \\
\hline 2000 & 57 & 2912 & 7,5 & 2,8 & 7,0 & 2,2 & 1,0 & 2,3 & 10 \\
\hline
\end{tabular}

$H=I N H, R=R M P, S=S M, E=E M B, Z=P Z A * M$. bovis wurde nicht gezählt

boviner Stämme für diese Auswertung vorliegen. Bei den Mehrfachresistenzen zeigt sich im Vergleich zu den Vorjahren ein Anstieg, bei allerdings weiterhin kleinen Fallzahlen. Es ist anzunehmen, dass es sich bei den Mehrfachresistenzen vorwiegend um sekundäre Resistenzen handelt. Daten zur Vorbehandlung der Patienten stehen jedoch nicht zur Verfügung $[1,2,7]$.

Die Daten des Nationalen Referenzzentrums (NRZ) für Mykobakterien in Borstel zeigten 2000 bei 2198 untersuchten Stämmen deutlich höhere Resistenzraten (INH: 15,4\%; SM: 13,7\%; RMP: 5,8\%; EMB: 4,3\%; PZA: 2,3\% sowie INH+RMP: 5,5\% und jegliche Resistenz: 19,4\%). Da in Borstel aber überdurchschnittlich viele Stämme von Patienten eingehen, bei denen eine therapeutische Problematik besteht, sind diese Ergebnisse nicht als repräsentativ für Deutschland anzusehen. Darüber hinaus beziehen sich diese Angaben nicht auf Neuerkrankungen, sondern auf prävalente Fälle.

\section{Behandlungsergebnisse}

In der DZK-Studie konnten ebenfalls die Behandlungsergebnisse ermittelt werden (Abb.14). Insgesamt konnte bei allen Patientengruppen ein Therapieerfolg in 74,2\% verzeichnet werden. Allerdings sind darunter 5,5\%, über die keine Angaben erhältlich waren (besonders jüngere mobile Patienten). Während $4,7 \%$ an

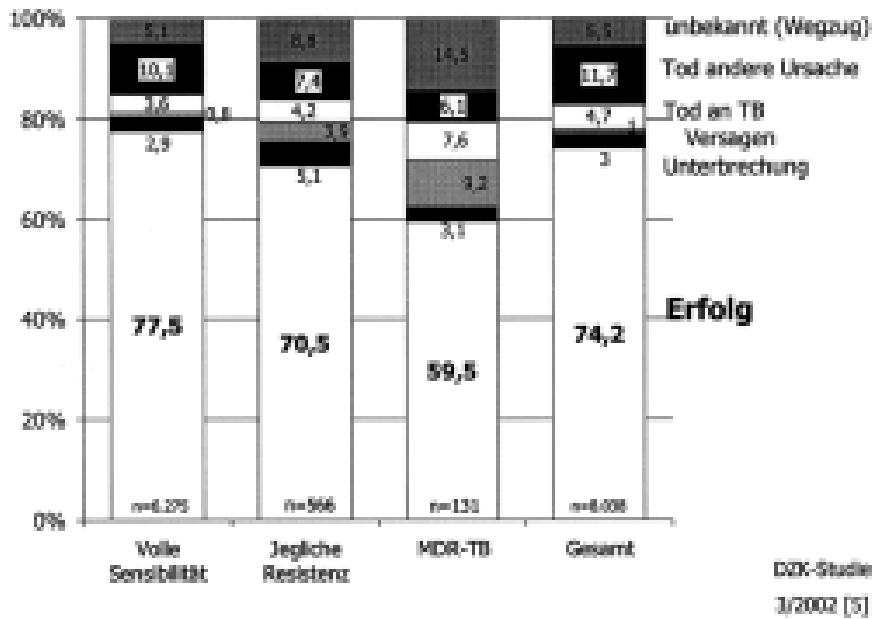

Abb. 14 Behandlungsergebnisse (\%) bei Tuberkulose nach Resistenz, $1997-2000$.

TB verstarben, starben weitere $11,7 \%$ durch andere Ursachen (vor allem ältere Patienten). Diese Einflussfaktoren verzerren die Ergebnisse des Therapieerfolges und führen dazu, dass das von der WHO propagierte Ziel von 85\% Behandlungserfolg in Deutschland nicht erreicht wird. 
Bei Patienten mit einer multiresistenten Tuberkulose wurde in 59,5\% der Fälle die Therapie erfolgreich abgeschlossen. Bei 9,2\% versagte die Therapie, 7,6\% starben an Tuberkulose, 6,1\% starben durch andere Ursachen, 3,1\% unterbrachen ihre Tuberkulosetherapie und von 14,5\% der Patienten konnten keine Angaben ermittelt werden. Es wird deutlich, dass der Therapieerfolg und die Mortalität entscheidend von der Resistenzlage und dem Alter beeinflusst werden.

\section{Zusammenfassung}

Die epidemiologische Situation der Tuberkuloseerkrankungen in Deutschland ist unverändert stabil. Die Inzidenz für 2000 betrug 11,0 pro 100000 Einwohner und ist somit weiterhin rückläufig. Die 2000 gemeldeten Tuberkulosefälle (alle Formen) zeigten im Vergleich zu 1999 einen Rückgang um 9,1\%. Der Rückgang an offener Tuberkulose betrug 8,2\%, an geschlossener Tuberkulose $11,5 \%$ sowie $8,8 \%$ bei der extrapulmonalen Tuberkulose. Der Ausländeranteil betrug, ähnlich wie im Vorjahr, 33,6\%, bei den Kindertuberkulosen $60,8 \%$. Die Zahl der Tuberkuloseerkrankungen bei Kindern stieg 2000, nach einem deutlichen Rückgang 1999 $(-13,3 \%)$, erneut an $(+4,7 \%)$. Das Erkrankungsrisiko ausländischer Kinder lag im Vergleich zu dem einheimischer Kinder durchschnittlich um das 7-19fache höher. Unverändert zeigte sich eine höhere Inzidenz im Alter; die ausländische Bevölkerung wies drei Altersgipfel auf.

Unter Berücksichtigung der dargestellten Daten zur Resistenzentwicklung, welche die derzeitige Problematik in den Herkunftsländern widerspiegeln, muss auch weiterhin von einer langsamen Zunahme der Resistenzen in Deutschland ausgegangen werden, wobei hier vor allem ausländische Bevölkerungsgruppen betroffen sind. Insbesondere die Zunahme der (Multi-)Resistenzen und die rapide Zunahme der HIV-Epidemie in den NUS sind auch für die Bevölkerung Deutschlands von Bedeutung [10]. Eine Eindämmung der dortigen Tuberkulose- und HIVEpidemie liegt somit im eigenen Interesse. Hier ist vor allem eine stärkere deutsche Beteiligung an internationalen Tuberkuloseund HIV-Kontrollmaßnahmen vor Ort erforderlich. Unter Berücksichtigung der Verschlechterung der Resistenzlage ist auf die zwingende Therapieeinleitung mit mindestens vier Antituberkulotika bei Neuerkrankungen dringend hinzuweisen [15].

Bei abnehmendem Erkrankungsrisiko in der Allgemeinbevölkerung konzentrieren sich in Deutschland die Tuberkulosekontrollmaßnahmen zunehmend auf Risikopopulationen. Die effektive Kontrolle und effiziente Bekämpfung der Tuberkulose erfordert unter anderem die Identifikation gefährdeter Personengruppen, die konsequente Durchführung von Umgebungsuntersuchungen und die damit verbundenen erforderlichen Präventionsmaßnahmen [9]. Effektive, kostengünstige Prävention und Therapie der Tuberkulose setzen die Kenntnis aktueller epidemiologischer Trends und somit eine fundierte Datengrundlage zwingend voraus. Hierfür schafft die Umsetzung des Infektionsschutzgesetzes eine wichtige Grundlage.

\section{Literatur}

${ }^{1}$ Deutsches Zentralkomitee zur Bekämpfung der Tuberkulose. 27. Informationsbericht 2002. Frankfurt/Main: pmi Verlagsgruppe (erhältlich beim DZK),

${ }^{2}$ Deutsches Zentralkomitee zur Bekämpfung der Tuberkulose. 26. Informationsbericht 2000. Frankfurt/Main: pmi Verlagsgruppe (erhältlich beim DZK),

${ }^{3}$ Deutsches Zentralkomitee zur Bekämpfung der Tuberkulose. Richtlinien zur medikamentösen Behandlung der Tuberkulose im Erwachsenen- und Kindesalter. Pneumologie 2001; 55: 494-511

${ }^{4}$ Espinal MA, Laszlo A, Simonsen L et al. Global trends in resistance to antituberculosis drugs. N Engl J Med 2001; 344: 1294-1303

${ }^{5}$ Forßbohm M. Studie des DZK zur Epidemiologie der Tuberkulose Zwischenbericht 2000. In: Deutsches Zentralkomitee zur Bekämpfung der Tuberkulose: 27. Informationsbericht 2002. Frankfurt/Main: pmi Verlagsgruppe

${ }^{6}$ Iseman MD. Treatment of multidrug-resistant tuberculosis. N Engl J Med 1993; 329: $784-791$

${ }^{7}$ Küchler R (Auswertung), Müller-Brundaler U, Urbanczik R. Korrespondenz an Dr. R. Küchler, Krankenhaus Neukölln, Rudower Str. 98, 12351 Berlin

${ }^{8}$ Loddenkemper R, Hauer B, Sagebiel D et al. Tuberkuloseepidemiologie in Deutschland und der Welt mit Schwerpunkt Osteuropa. Bundesgesundheitsblatt 1999; 42: $683-693$

${ }^{9}$ Loddenkemper $\mathrm{R}$. The need for antituberculosis drug resistance surveillance in Europe (Editorial). Eur Respir J 2000; 16: 195 - 196

10 Loddenkemper R, Sagebiel D, Brendel A. Strategies against multidrugresistant tuberculosis. ERJ Supplement „Respiratory Infections and Tuberculosis“. Eur Respir J 2002; 20: 1-13

11 Perelman MI. Tuberculosis in Russia. Int J Tuberc Lung Dis 2000; 4 $1097-1103$

12 Robert Koch-Institut. Ständige Impfkommission am Robert Koch-Institut (STIKO): Impfempfehlungen. Epidemiologisches Bulletin 1998; 15: $109-112$

${ }^{13}$ Robert Koch-Institut. Zur Struktur der Tuberkulosemorbidität in Deutschland - Ergebnisse der Studie des DZK zur Epidemiologie der Tuberkulose. Epidemiologisches Bulletin 1998; 49: 349-351

${ }^{14}$ Sagebiel D, Brendel A, Forßbohm M et al. Gefährliche Zunahme multiresistenter Tuberkulosen in der ehemaligen Sowjetunion. Pneumologie 2002; 56: $7-10$

15 Statistisches Bundesamt Wiesbaden. Abteilung VII B-176

${ }^{16}$ Statistisches Jahrbuch 2001 für die Bundesrepublik Deutschland. Stuttgart: Metzler-Poeschel Verlag,

17 UNAIDS 2001. AIDS epidemic update, December 2001. UNAIDS/01.74E - WHO/CDS/CSR/NCS/2001

${ }^{18}$ WHO Report 2002. Global Tuberculosis Control. WHO/CDS/ TB2002.295

${ }^{19}$ WHO/IUATLD. Antituberculosis drug resistance in the world. Report No. 2. Prevalence and trends. WHO/CDS/TB/2000.278 